\title{
Proposta de uma modelagem computacional para propagação de trincas em vigas de pontes sob carregamento cíclico
}

\author{
Proposal for a computational modeling for crack propagation in bridge beams under cyclic loading \\ Propuesta de modelado computacional para la propagación de fisuras en vigas de puentes bajo \\ carga cíclica
}

Recebido: 08/03/2021 | Revisado: 14/03/2021 | Aceito: 19/03/2021 | Publicado: 27/03/2021

\author{
Rafael Leandro Fernandes Melo \\ ORCID: https://orcid.org/ 0000-0003-4422-2206 \\ Universidade Federal do Ceará, Brasil \\ E-mail: rafaellfmelo@gmail.com \\ Adroaldo José Silva de Moura Filho \\ ORCID: https://orcid.org/ 0000-0002-8324-8524 \\ Universidade Federal do Ceará, Brasil \\ E-mail: adroaldo_1@msn.com \\ Marcos Fábio Veríssimo Montezuma \\ ORCID: https://orcid.org/ 0000-0002-8669-0422 \\ Universidade Federal do Ceará, Brasil \\ E-mail: marcosmontezuma@ufc.alu.br \\ Enio Pontes de Deus \\ ORCID: https://orcid.org/ 0000-0002-3933-0803 \\ Universidade Federal do Ceará, Brasil \\ E-mail: epontes@ufc.br
}

\begin{abstract}
Resumo
Pontes de aço normalmente estão sujeitas a carregamentos cíclicos de amplitude variável com tipo e frequência de tráfego ao acaso. As pontes de aço, por serem estruturas de comportamento dinâmico, estão sujeitas a falhar por processo de fadiga. Determinando a localização e a amplitude de uma falha existente, tem-se de imediato a preocupação com a previsão de propagação da mesma a fim de ter-se um tempo conveniente de reparo. Este trabalho avaliou-se o problema de propagação de trincas de fadiga em vigas de pontes de aço sob diferentes espectros de carregamentos cíclicos de amplitude variável. Foi desenvolvido um programa computacional para a análise do crescimento das trincas utilizando-se os modelos do Valor Médio Quadrático (Root Mean Square - RMS) e Ciclo-aCiclo. Considerou-se como variáveis de entrada do programa diferentes tamanhos iniciais de trincas e diferentes histórias de carregamentos. Com isso, avaliou-se a dispersão dos resultados de vida esperada da estrutura com a variação dos diferentes parâmetros de entrada.
\end{abstract}

Palavras-chave: Pontes; Carregamento cíclico; Propagação de trincas.

\begin{abstract}
Steel bridges are normally subjected to random loads with different traffic frequencies. This way, steel bridges are structures of dynamic behavior and are subjected to fatigue failure process. After localizing and determine the amplitude of an existing flaw, its important predicts crack propagation and the convenient repair time. At this work, the fatigue crack propagation on steel beam bridges, has been studied under different spectrum of variable-amplitude loading. To study the fatigue crack growth was developed a computational code, using the Root Mean Square (RMS) and the Cycle-by-Cycle models. Different loads histories and initial crack length were considered input variables. This way was evaluating the dispersion of results of the expected structural life choosing different initial parameters.
\end{abstract}

Keywords: Bridges; Cyclic loading; Crack propagation.

\section{Resumen}

Los puentes de acero suelen estar sujetos a cargas cíclicas de amplitud variable con tipos y frecuencias de tráfico aleatorios. Los puentes de acero, por ser estructuras de comportamiento dinámico, están sujetos a fallas por fatiga. Al determinar la ubicación y la amplitud de una falla existente, existe una preocupación inmediata con el pronóstico de su propagación para tener un tiempo de reparación conveniente. Este trabajo evaluó el problema de la propagación de fisuras por fatiga en vigas de puentes de acero bajo diferentes espectros de cargas cíclicas de amplitud variable. Se desarrolló un programa de computadora para analizar el crecimiento de grietas utilizando los modelos Root Mean Square (RMS) y Cycle-by-Cycle. Se consideraron como variables de entrada del programa diferentes tamaños 
iniciales de grietas y diferentes historiales de carga. Con esto, se evaluó la dispersión de los resultados de vida esperados de la estructura con la variación de los diferentes parámetros de entrada.

Palabras clave: Puentes; Carga cíclica; Propagación del crack.

\section{Introdução}

Pontes de aço normalmente estão sujeitas a carregamentos cíclicos de amplitude variável com tipo e frequência de tráfego ao acaso (Deus, 1997; Garcez et al., 2012; Ding et al, 2021). Devido a defeitos como trincas, porosidades, inclusões, defeitos de soldagem e pormenores inadequados inerentes ao material, ao processo de fabricação ou ao projeto, podem ser produzidas grandes concentrações de tensões nestes locais e consequentemente o processo de fadiga pode iniciar-se até mesmo numa fase bastante inicial de uso da estrutura (Dantas, 2010; Arias, 2015).

Atualmente, os materiais empregados para fabricação de pontes de aço e elementos estruturais são aços de alta resistência e baixo teor de elemento de liga (aços estruturais), que tentam aliar as propriedades de resistência mecânica a uma boa tenacidade à fratura do material (Haghani \& Yang, 2016; Wang, 2020).

Mesmo assim, elementos de pontes de aço tendem a ser sensíveis ao processo de fadiga (Kwon \& Frangopol, 2010). Os materiais de alta resistência mecânica têm baixa resistência às trincas, e como consequência, a resistência remanescente deles em presença de trincas e defeitos é pequena (Manhart, 2000; Terasawa et al 2021). Uma trinca formada em qualquer elemento estrutural da ponte introduz uma variação de rigidez local que muda o seu comportamento dinâmico, comprometendo toda a estrutura (Law \& Zhu, 2004).

Com isso observamos que as pontes de aço, por serem estruturas de comportamento dinâmico, estão sujeitas a falhar por processo de fadiga. Estas estruturas podem fraturar de maneira catastrófica sob tensões abaixo das tensões mais elevadas para as quais foram projetadas causando, assim, grande prejuízo econômico e risco às vidas humanas (Chen \& Wu, 2010; Pu et al 2021; Lu et al 2021). As principais causas que ocasionam o problema de fadiga em pontes de aço são:

1. Idade da estrutura;

2. Carregamentos alternados;

3. Tipos de detalhes estruturais concentradores de tensão;

4. Frequência de tráfego com amplitude variável;

5. Defeitos do material;

6. Baixa tenacidade à fratura do material;

7. Defeitos de soldagem;

8. Defeitos de projeto.

Diversos autores têm usado métodos numéricos para simular e prever o comportamento de trincas sujeitas a carregamentos variáveis (Skorupa, 1998; Kujawski, 2001; Huang, Torgeir \& Cui, 2008; Grzeskowiak et al 2021). Dentre os principais modelos de propagação de trincas mais utilizados na literatura destacam-se os modelos do Valor Médio Quadrático (Root Mean Square - RMS) e o modelo Ciclo-a-Ciclo. O estudo de propagações de trincas sob carregamentos variáveis requer conhecimentos nas áreas de fadiga e mecânica da fratura.

Neste trabalho foi desenvolvido um modelo numérico computacional que considera a propagação de trincas no caso específico de vigas de pontes de aço. São acrescentadas ao modelo, como dados de entrada, diversas histórias de carregamentos de amplitude variável obtidas através de simulação numérica. São analisados carregamentos de amplitude variável estacionários e não-estacionários. Os objetivos principais do estudo são:

1. Desenvolver uma metodologia para simular, usando o modelo computacional desenvolvido, o comportamento de propagação de trincas de tamanhos iniciais diferentes quando sujeitas a diversos espectros de carregamentos;

2. Analisar a metodologia dos métodos numéricos Valor Médio Quadrático (Root Mean Square - RMS) e Ciclo-a- 
Ciclo.

\section{Metodologia}

Essa pesquisa trata-se de uma metodologia computacional qualitativa (Pereira et al, 2018). Para o desenvolvimento da modelagem da propagação de trincas em uma ponte, foi proposta a seguinte metodologia: definir o modelo da ponte, definir o modelo de propagação de trinca na ponte, os materiais da ponte, modelar a viga, modelar a viga trincada, definir as regras de propagação da trinca, definir os blocos de carregamento, e realizar os modelos Valor Médio Quadrático (Root Mean Square RMS) e Ciclo-a-Ciclo.

Os programas utilizados para o desenvolvimento da modelagem foram: G_CAVR, ViDa, DELTA_P_rms e CICLO_A_CICLO.

\subsection{Projeto de Ponte Modelo (AASHTO)}

Para o desenvolvimento do projeto, foi considera o modelo da norma américa AASHTO. Essa, define membros planos de pontes em redundantes e não-redundantes. Redundantes são aqueles que se falharem não causam o colapso da estrutura pois as cargas são redistribuídas para membros adjacentes e caminhos alternativos, o que não ocorre com os não redundantes, pois se falharem em serviço causam o colapso da ponte. No presente trabalho a viga é considerada como nãoredundante.

No projeto de uma ponte modelo não-trincada, utilizou-se o exemplo de uma ponte biapoiada com vão de $24.000 \mathrm{~mm}$. A viga consiste em um perfil soldado em I, onde a mesa superior tem dimensão de $360 \mathrm{~mm}$ e a mesa inferior $750 \mathrm{~mm}$. A alma tem altura total de $1.356 \mathrm{~mm}$. Suas propriedades geométricas são mostradas abaixo, na Figura 1 e Tabela 1.

Figura 1 - Propriedades geométricas da viga.

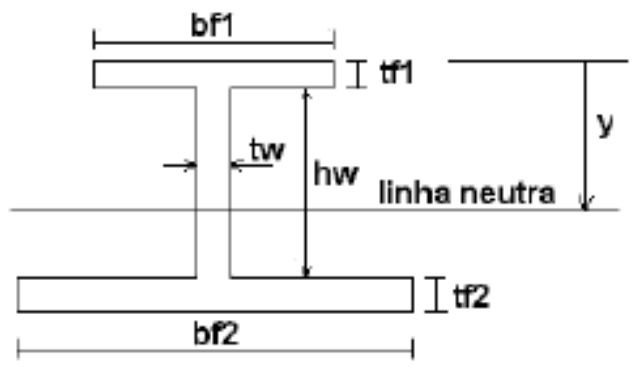

Fonte: Autores (2021).

Tabela 1 - Propriedades geométricas da viga.

\begin{tabular}{llll}
\hline \multicolumn{3}{c}{ VI 1400 } \\
\hline Mesa superior & tf1 $=19 \mathrm{~mm}$ & bf1 $=360 \mathrm{~mm}$ & Área $=6.840 \mathrm{~mm}^{2}$ \\
Alma & $\mathrm{tw}=10 \mathrm{~mm}$ & $\mathrm{hw}=1.356 \mathrm{~mm}$ & Área $=13.560 \mathrm{~mm}^{2}$ \\
Mesa inferior & $\mathrm{tf} 2=25 \mathrm{~mm}$ & bf2 $=750 \mathrm{~mm}$ & Área $=18.750 \mathrm{~mm}^{2}$ \\
\hline
\end{tabular}

Fonte: Autores (2021).

Onde,

Área Total $=39.150 \mathrm{~mm}^{2}$

$\mathrm{L}=24.000 \mathrm{~mm}$ 
Centro de gravidade $\mathrm{y}=907,6 \mathrm{~mm}$

Momento de Inércia $\mathrm{I}=0,119 \times 10^{11} \mathrm{~mm}^{4}$

Módulo de elasticidade $\mathrm{E}=200 \mathrm{GPa}$

Limite de escoamento se $=276 \mathrm{MPa}$

Peso próprio $\mathrm{Pp}=72,36 \mathrm{kN}$

\subsection{Verificação de Fadiga (AASHTO)}

A norma americana AASHTO estabelece os limites necessários para a verificação de fadiga em estruturas redundantes e não-redundantes. Tais estruturas quando sujeitas a variações repetidas ou reversas de tensão devem ser projetadas de acordo com as tensões admissíveis para ciclos e categorias, segundo os detalhes construtivos. A faixa de tensão Fr é definida como a diferença algébrica entre a máxima e a mínima tensão $\left(\mathrm{s}_{\max }-\mathrm{s}_{\min }\right)$.

A Tabela 2 mostra os valores de tensões admissíveis e os respectivos números de ciclos para estruturas nãoredundantes. A viga em estudo está classificada na Categoria B pois se aplica a uma grande variedade de detalhes em vigas soldadas, como as soldas de entalhe (groove weld) em ligação de topo, localizadas na alma e nas mesas das vigas I.

Tabela 2 - Limites de tensão de fadiga admissíveis para categoria B - Estruturas não-redundantes (AASHTO).

\begin{tabular}{ccccc}
\hline \multicolumn{5}{c}{ Estruturas Não-redundantes - $\mathrm{F}_{\mathrm{r}}(\mathrm{MPa})}$, \\
\hline CATEGORIA & $\begin{array}{r}100.000 \\
\text { CICLOS }\end{array}$ & $\begin{array}{c}500.000 \\
\text { CICLOS }\end{array}$ & $\begin{array}{c}2.000 .000 \\
\text { CICLOS }\end{array}$ & $\begin{array}{c}\text { ACIMA DE } \\
2.000 .000 \\
\text { CICLOS }\end{array}$ \\
\hline B & 269 & 159 & 110 & 110 \\
\hline
\end{tabular}

Fonte: Autores (2021).

Foi considerado um trem tipo classe 45 - NBR 7188/84 na qual a base do sistema é um veículo de $450 \mathrm{kN}$ de peso total e coeficiente de impacto igual a 1,26 (NBR 7187/87) calculou-se a tensão máxima na mesa inferior levando-se em consideração que a ponte seria formada por duas vigas iguais e a carga pode assim ser dividida em $225 \mathrm{kN}$ para cada viga. Neste caso a ponte é considerada de classe 45 . O resultado é mostrado na Eq 1.

$$
\sigma_{r}=\frac{M_{m}}{W_{m}}=\frac{1.701}{0,02419}=70,32 M P a<F_{r}
$$

(acima de 2.000.000 ciclos)

Onde,

$\mathrm{W}_{\mathrm{m}}$ é o módulo de resistência da seção $\left(\mathrm{m}^{3}\right)$;

$\mathrm{M}_{\mathrm{m}}$ é o momento proveniente da carga móvel (kN.m).

\subsection{Materiais para Vigas de Aço}

A Tabela 3 mostra os aços reconhecidos pela AASHTO para estruturas de pontes com as propriedades mínimas de resistência à tração e limite de escoamento. O módulo de elasticidade mínimo de todas as grades de aços estruturais é assumido como sendo igual a $200 \mathrm{GPa}$. 
Research, Society and Development, v. 10, n. 3, e57510313670, 2021

(CC BY 4.0) | ISSN 2525-3409 | DOI: http://dx.doi.org/10.33448/rsd-v10i3.13670

Tabela 3 - Propriedades mínimas de resistência de aços estruturais (AASHTO).

\begin{tabular}{|c|c|c|c|c|c|}
\hline Tipo & $\begin{array}{c}\text { Aço } \\
\text { Estrutural }\end{array}$ & \multicolumn{2}{|c|}{$\begin{array}{c}\text { Aço Baixa-Liga Alta } \\
\text { Resistência }\end{array}$} & $\begin{array}{l}\text { Acco Baixa-Liga } \\
\text { Temperado e } \\
\text { Revenido }\end{array}$ & $\begin{array}{c}\text { Aço Liga de Alto Limite } \\
\text { de Escoamento } \\
\text { Temperado e Revenido }\end{array}$ \\
\hline Designaçào & M270 & $M 270$ & M 270 & M 270 & $M 270$ \\
\hline AASHTO & Gr. 36 & Gr. 50 & Gr. $50 \mathrm{~W}$ & Gr. 70W & Gr. $100 / 100 \mathrm{~W}$ \\
\hline Equivalente & A 709 & A 709 & A 709 & A 709 & A 709 \\
\hline ASTM & A 36 & Gr. 50 & Gr. $50 \mathrm{~W}$ & Gr. $70 \mathrm{~W}$ & Gr. $100 / 100 \mathrm{~W}$ \\
\hline Res.Traçào (min.) & $400 \mathrm{MPa}$ & $448 \mathrm{MPa}$ & $482 \mathrm{MPa}$ & $620 \mathrm{Mpa}$ & $758 \mathrm{MPa}$ \\
\hline Res. Esc. (min.) & $248 \mathrm{MPa}$ & $344 \mathrm{MPa}$ & $344 \mathrm{MPa}$ & $482 \mathrm{Mpa}$ & $689 \mathrm{MPa}$ \\
\hline
\end{tabular}

Fonte: Autores (2021).

Neste trabalho consideramos o material da viga como sendo o aço A-36 que tem microestrutura ferrítica perlítica. O fator de intensidade de tensão crítico e o limite se escoamento do aço A-36 são mostrados nas Eq. 2 e Eq. 3 :

$$
\begin{aligned}
& K_{I C}=55 \mathrm{MPa} \sqrt{\mathrm{m}} \\
& \sigma_{\text {e }}=276 \mathrm{MPa}
\end{aligned}
$$

\subsection{Modelagem 3D da Viga para Estimar os Limites Máximo e Mínimo da Tensão de Serviço sem a Presença de} Trincas

Para se estimar os limites máximo e mínimo da tensão de serviço na mesa inferior da viga considerou-se a carga mínima como sendo o peso próprio da estrutura, e a carga máxima como sendo o peso próprio mais o peso do veículo-tipo classe 45 com o fator de impacto (carga dinâmica). Como mostrados na Eq. 4 e Eq. 5.

$$
\begin{aligned}
& P_{\min }=72,36 \mathrm{kN} \\
& P_{\text {máx }}=72,36+283,5=355,86 \mathrm{kN}
\end{aligned}
$$

Logo, todos os carregamentos aplicados no modelo da viga para análise de propagação das trincas deverão estar dentro destes limites. O programa ANSYS é utilizado aqui como ferramenta para a análise de tensões na viga I - V1. A viga é modelada de acordo com as dimensões e propriedades mecânicas especificadas com o elemento finito SHELL43 e os carregamentos mínimo e máximo são aplicados no modelo. Para título de arredondamento consideramos $\mathrm{P}_{\min }=70,0 \mathrm{kN}$ e $\mathrm{P}_{\max }$ $=360,0 \mathrm{kN}$. As Figuras 2 e 3 mostram respectivamente as duas situações. 
Research, Society and Development, v. 10, n. 3, e57510313670, 2021

(CC BY 4.0) | ISSN 2525-3409 | DOI: http://dx.doi.org/10.33448/rsd-v10i3.13670

Figura 2 - Modelagem da Viga I -V1 no programa ANSYS para situação de carregamento mínimo ( $\mathrm{P}=70 \mathrm{kN})$.

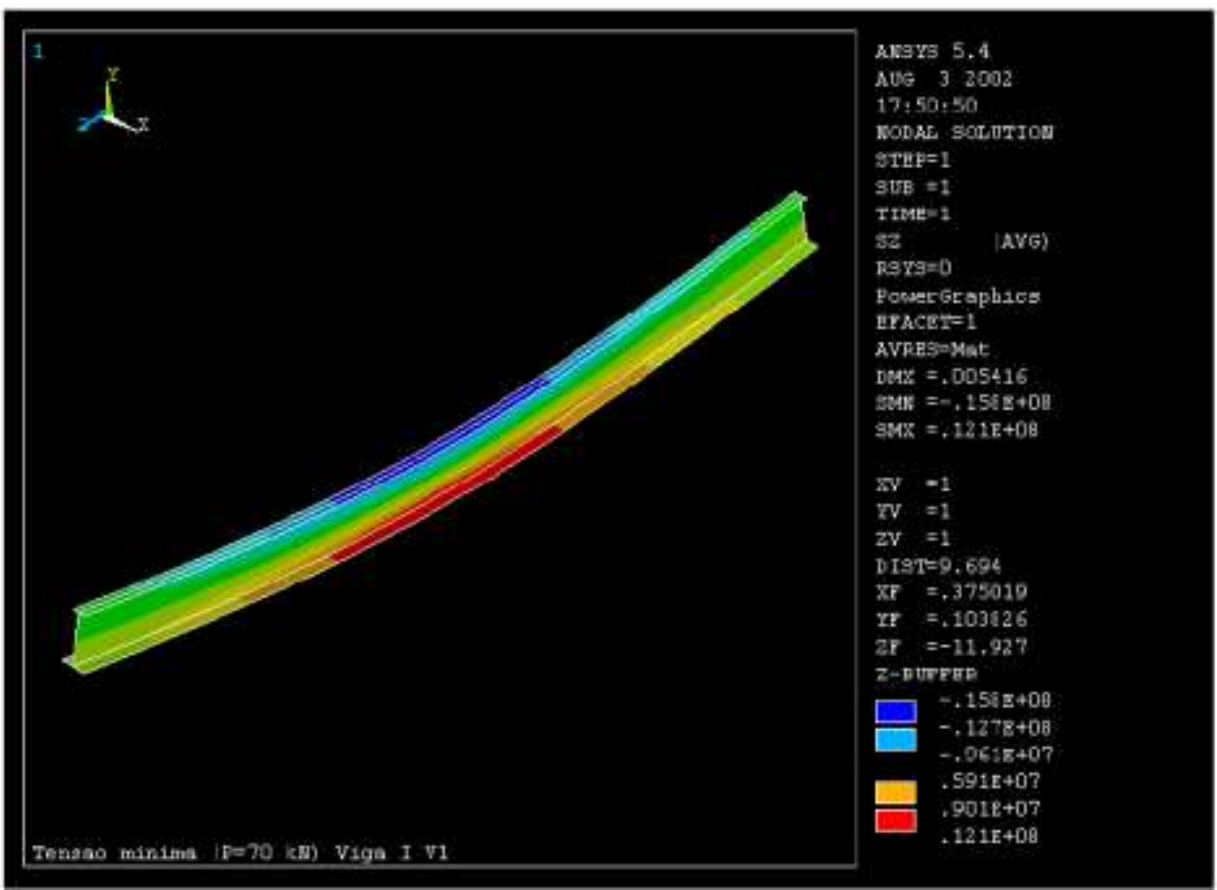

Fonte: Autores (2021).

Figura 3 - Modelagem da Viga I -V1 no programa ANSYS para situação de carregamento máximo $(\mathrm{P}=360 \mathrm{kN})$.

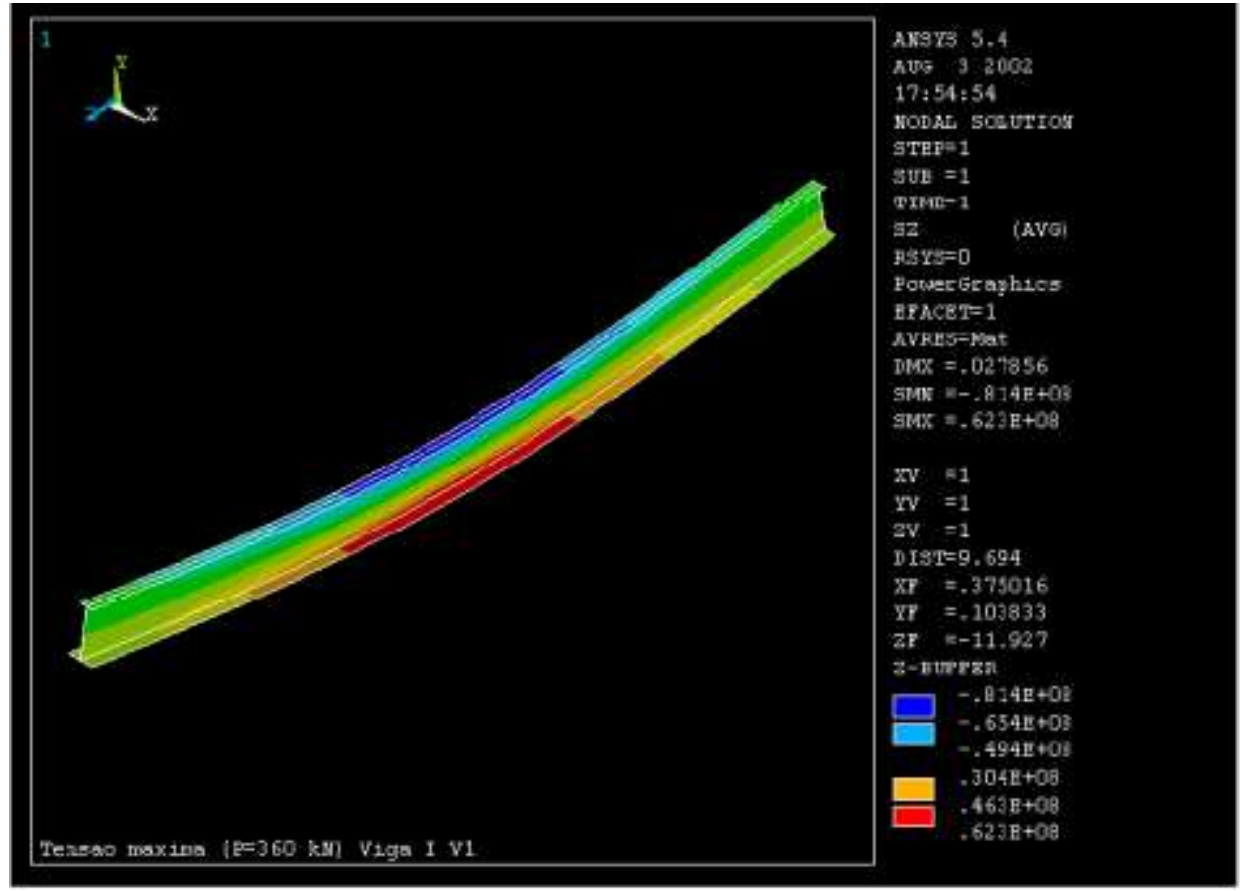

Fonte: Autores (2021).

De acordo com os resultados da modelagem observamos que os limites de tensões para uma viga sem trinca foram:

$$
\begin{array}{ll}
\sigma_{p r \min }=12,1 \mathrm{MPa} & \text { (tensão mínima de projeto) } \\
\sigma_{p r \max }=62,3 \mathrm{MPa} & \text { (tensão máxima de projeto) }
\end{array}
$$


$\Delta \sigma_{p r}=50,12 \mathrm{MPa}$

Observamos que a viga trabalha perfeitamente dentro do regime elástico do material quando não há presença de defeitos.

\subsection{Modelo de Viga Trincada}

Verificou-se no que todos os requisitos da AASHTO para o projeto da ponte são satisfeitos, mas deve-se observar que está se adequa ao projeto de novas estruturas e não leva em consideração a presença de defeitos encontrados posteriormente. Neste modelo assume-se inicialmente uma trinca de comprimento ao em Modo I de abertura na alma da viga transpassando a mesa inferior e posicionada na metade do comprimento do vão, como mostra a Figura 3. O processo de fadiga e crescimento da trinca se desenvolverá na viga devido a um carregamento $\mathrm{P}$ de amplitude variável. Devemos observar que neste modelo não se leva em consideração as tensões residuais na região da solda; isto porque assume-se que a trinca se originou como uma pequena fissura ou defeito na região da solda, levou um determinado tempo para crescer e encontra-se num estágio avançado onde já possui um tamanho bastante considerável na alma da viga e fora da influência de tensões residuais. Deseja-se então prever o tempo de vida da estrutura para um reparo conveniente.

Figura 4 - Modelo de viga biapoiada trincada em Modo I.

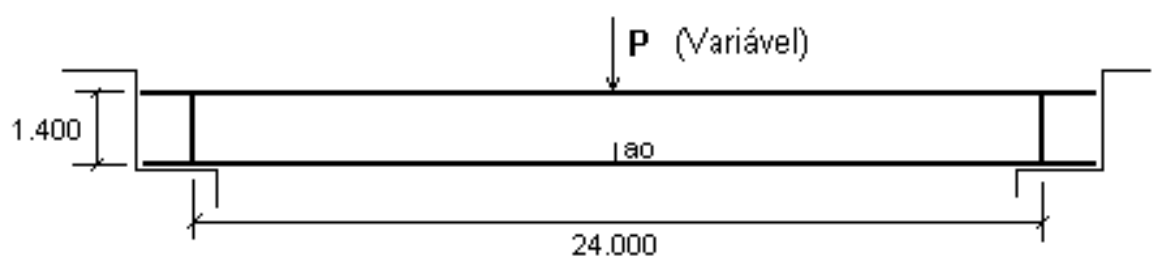

Fonte: Autores (2021).

\subsection{Regras de Propagação de Trincas Por Fadiga}

O presente trabalho utiliza como regra de propagação de trincas a regra de Paris Erdogan. Esta regra correlaciona a taxa de crescimento da trinca com a variação do fator de intensidade de tensão, como mostrado na Eq. 6:

$$
\frac{d A}{d N}=A \cdot \Delta K^{m}
$$

Onde,

A e m são constantes do material.

As estimativas de dados reais citadas por Komarovsky \& Haslach (2003) indicam que as taxas de crescimento de trinca por fadiga para os aços ferrítico perlíticos podem ser calculadas com base na Eq. 7:

$$
\frac{d A}{d N}=6,9 \times 10^{-12}\left(\Delta K^{3}\right)\left(\frac{m}{\text { ciclo }}\right) e \Delta K(M P a \sqrt{m})
$$

Para se estimar a vida de fadiga sob carregamento de amplitude variável são utilizados os métodos de crescimento pelo valor médio quadrático RMS (root-mean-square) e ciclo-a-ciclo. 


\subsection{Blocos de carregamento}

Os carregamentos utilizados nas simulações de propagação das trincas foram divididos em 7 Blocos de 10 carregamentos, caracterizados da seguinte forma:

Os carregamentos dos Blocos 1, 2, 3 e 4 são denominados de Carregamentos de Amplitude Variável Estacionários (CAVE) e são seqüenciados de CAVE1 a CAVE40. Caracterizam-se pela repetição dos ciclos de carregamentos ao longo do tempo.

Os carregamentos do Bloco 5 são denominados de Carregamentos de Amplitude Variável Randômicos e caracterizam-se pela variação aleatória da amplitude do carregamento com o tempo.

Os carregamentos do Bloco 6 são denominados de Carregamentos de Amplitude Variável Randômicos. Da mesma maneira que nos carregamentos do Bloco 5, estes também se caracterizam pela variação aleatória da amplitude do carregamento com

o tempo. A diferença principal em relação ao Bloco 5 é que nestes a amplitude de carregamentos não varia do primeiro ao último carregamento do bloco, variando somente o grau de aleatoriedade entre eles.

Os carregamentos do Bloco 7 são denominados também de Carregamentos de Amplitude Variável Randômicos. Caracterizam-se por terem amplitudes de carregamentos que variam randomicamente com o tempo e por possuírem uma carga mínima constante, esta chamada de peso próprio. As amplitudes são crescentes desde CAVE61 a CAVE70. Estes carregamentos têm uma particularidade especial por apresentarem uma curva de distribuição de probabilidade adequada para descrever o campo de carregamentos avaliados em pontes.

\subsection{Modelo de Crescimento de Trincas Através do Método do Valor Médio Quadrático RMS (Root-Mean-Square)}

Podemos tratar o problema de previsão da vida à fadiga de uma peça sujeita a um carregamento de amplitude variável substituindo este por um carregamento de amplitude constante que lhe seja equivalente, no sentido de causar o mesmo crescimento de trinca. Então o valor de $\Delta K$ pode ser calculado a partir dos valores RMS dos picos e dos vales das tensões atuantes sobre a peça. Considerando que a parte negativa dos carregamentos deve ser desconsiderada, temos as Eq 8, 9 e 10:

$$
\begin{aligned}
& \sigma_{\max }=\sqrt{\frac{\sum_{i=1}^{p}\left(\sigma_{\max }\right)^{2}}{p}} \\
& \sigma_{\min }=\sqrt{\frac{\sum_{i=1}^{p}\left(\sigma_{\min }\right)^{2}}{p}} \\
& \Delta \sigma_{r \operatorname{ms}}=\sigma_{\max }-\sigma_{\min } \operatorname{com} \sigma_{\max }, \sigma_{\min } \geq 0
\end{aligned}
$$

Onde,

p e q são respectivamente o número de picos e vales do carregamento.

A previsão do número de ciclos que a trinca leva para crescer do comprimento inicial $a_{o}$ até o final $a_{f}$ é dada pela Eq. 11:

$$
N=\int_{a_{0}}^{a_{f}} \frac{d A}{f\left(\Delta K_{r m s}\right)}
$$

Vale ressaltar que o valor de $\Delta K_{r m s}$ de um carregamento variável é similar, mas não idêntico ao $\Delta K$ de um carregamento constante. Como toda estatística, $\Delta K_{r m s}$ não reconhece ordem temporal e não pode perceber alguns problemas como: 
1. Fratura súbita causada por um grande pico durante o carregamento variável $\left(K_{\max }=K_{c}\right)$

2. Interação entre ciclos ou efeito de retardo.

\subsection{Método de Crescimento de Trincas Ciclo-a-Ciclo}

A ideia básica deste método é associar a cada reversão do carregamento o crescimento que a trinca teria se apenas aquele meio ciclo atuasse sobre a peça. Sendo a taxa de propagação da trinca $\frac{d A}{d N}=f\left(\Delta K_{i}\right)$ e no i-ésimo $1 / 2$ meio ciclo do carregamento o comprimento da trinca é ${ }^{a_{i}}$, então a gama de tensão atuante é ${ }^{\Delta \sigma_{\bar{i}}}$ e o incremento da trinca ${ }^{\delta a_{\bar{i}}}$ pode ser dado pela seguinte expressão, Eq. 12 e 13:

$$
\begin{aligned}
\delta a_{i} & =\left(\frac{1}{2}\right) f\left(\Delta K_{i}\right) \\
\Delta K_{i} & =f\left(\frac{a_{i}}{W}\right) \Delta \sigma_{i} \sqrt{\pi a_{i}}
\end{aligned}
$$

onde $^{f\left(\frac{a_{i}}{W}\right)}$ é o fator que depende da geometria da peça.

$\mathrm{O}$ crescimento da trinca é quantificado pelo $\sum \delta a_{\bar{i}}$. Esta regra é similar em conceito ao acúmulo linear de dano.

Antes de quantificar o crescimento torna-se necessário reduzir a história de carregamento a uma sequência de eventos que possam ser estimados como compatíveis com dados de fadiga de amplitude constante. Os métodos que tornam tais reduções possíveis são conhecidas como métodos de contagem de ciclos.

Neste trabalho utilizaremos o método de contagem de ciclos denominado rain-flow. Este método utiliza um esquema específico de contagem de ciclos para estimar a ordem de tensões efetivas e identificar ciclos de tensões relacionados aos loops fechados de histerese na resposta tensão-deformação do material quando sujeito a um carregamento cíclico.

Este trabalho utiliza como ferramenta de contagem de ciclos o programa ViDa - Danômetro Visual. Neste programa utiliza-se a contagem de ciclos de forma sequenciada. Com esta técnica o efeito de cada grande evento é contado quando ele ocorre e não antes de sua ocorrência, como no método tradicional. A principal vantagem desta técnica é evitar a aplicação antecipada de sobrecargas, o que pode causar previsões não-conservativas.

Deve-se enfatizar que, ainda que o método de contagem rain-flow seja uma das mais efetivas ferramentas para prever vida de fadiga sob história de carregamento de amplitude variável, este não é capaz de descrever efeitos de interação de tensões ou efeitos de sequência.

A implementação de um algoritmo do método ciclo-a-ciclo não é numericamente difícil, mas requer muito esforço computacional. Tem as vantagens de garantir a inatividade da trinca quando num ciclo $D K_{i}<D K_{t h}$ e prever a fratura súbita causada por um grande pico durante o carregamento variável quando $K_{\max }=K_{\bar{c}}$.

\section{Resultados e Discussão}

O modelo computacional desenvolvido que utiliza o método do Valor Médio Quadrático para propagação de trincas, tem a seguinte sequência de utilização:

- Geração dos carregamentos variáveis através dos programas:

- G_CAVR, para carregamentos de amplitudes variáveis randômicos e 
- G_CAVE, para carregamentos de amplitudes variáveis estacionários;

- Contagem rain-flow dos carregamentos através do programa ViDa;

- Utilização do programa DELTA_P_rms para cálculo do valor de $\Delta$ Prms através de um arquivo de entrada de carregamentos na forma de Vales e Picos;

- Utilização do programa P_RMS para calcular o número de ciclos necessários para uma trinca crescer de um valor inicial ao até um valor final ac.

Já o modelo computacional desenvolvido com base no método Ciclo-a-Ciclo, tem a seguinte sequência de utilização:

- Geração dos carregamentos variáveis através dos programas:

- G_CAVR, para carregamentos de amplitudes variáveis randômicos e

- G_CAVE, para carregamentos de amplitudes variáveis estacionários;

- Contagem rain-flow dos carregamentos através do programa ViDa;

- Utilização do programa CICLO_A_CICLO para calcular o número de ciclos necessários para uma trinca crescer de um valor inicial ao até um valor final ac, através de um arquivo de entrada de carregamentos na forma de Vales e Picos.

Através da Figura 5 pode-se avaliar como ocorre a variação de $\Delta \sigma_{\text {rms }}$ desde o $1^{\circ}$ até o $10^{\circ}$ carregamento em cada bloco, desde o Bloco 1 até o Bloco 7.

Figura 5 - Variação de $\Delta \sigma_{\text {rms }}$ nos blocos de carregamentos 1 ao 7.

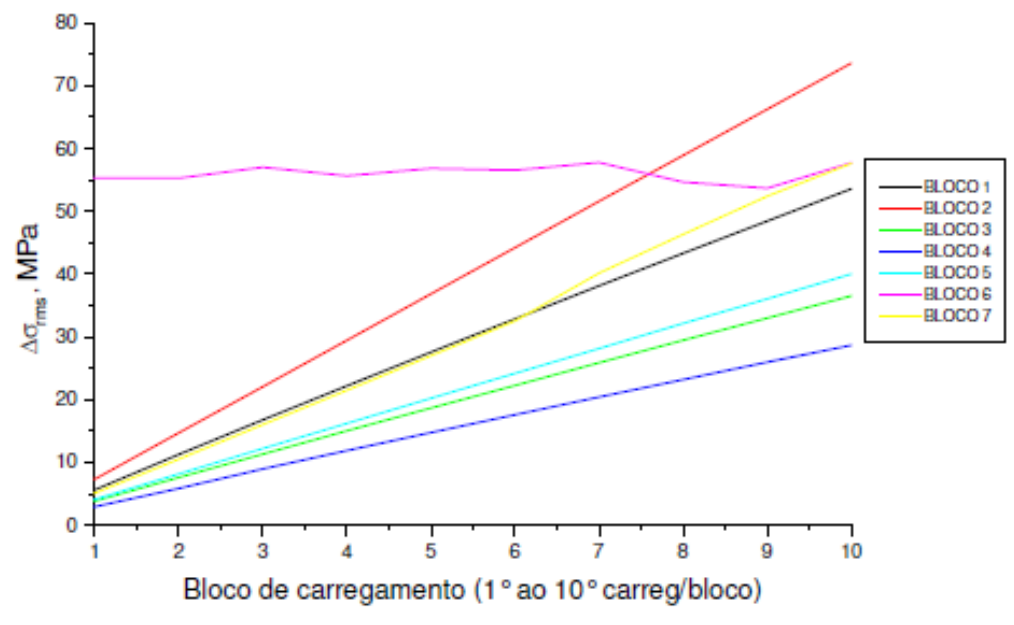

Fonte: Autores (2021).

\subsection{Resultados das Simulações com o Modelo do Valor Médio Quadrático (RMS)}

Esse trabalho utilizará os dados obtidos com o Bloco 1 e Bloco 6 na análise dos resultados, uma vez que na avaliação pelo modelo RMS pode-se considerar os carregamentos dos Blocos 1 e 7 como equivalentes, devido as diferenças entre as curvas de variação de ${ }^{D s_{r m s}}$ serem muito pequenas e o Bloco 6 corresponder a carregamentos onde a amplitudes de tensão estão na faixa de $50 \mathrm{MPa}$ a $60 \mathrm{MPa}$. Nas Figuras 6 e Figura 7 são apresentadas as curvas de crescimento das trincas de tamanho inicial ao até o valor crítico ${ }^{a_{\mathfrak{c}}}$ para o Bloco de carregamento 1 e 6 . Observou -se uma vantagem do modelo do Valor Médio Quadrático RMS para a construção dos gráficos, devido ao menor número de dados necessários para plotar as curvas e ao menor esforço computacional deste modelo em relação ao Ciclo-a-Ciclo. 
Figura 6 - Tamanho da trinca x Número de ciclos para diversos comprimentos iniciais de trincas - BLOCO 1 de carregamentos.

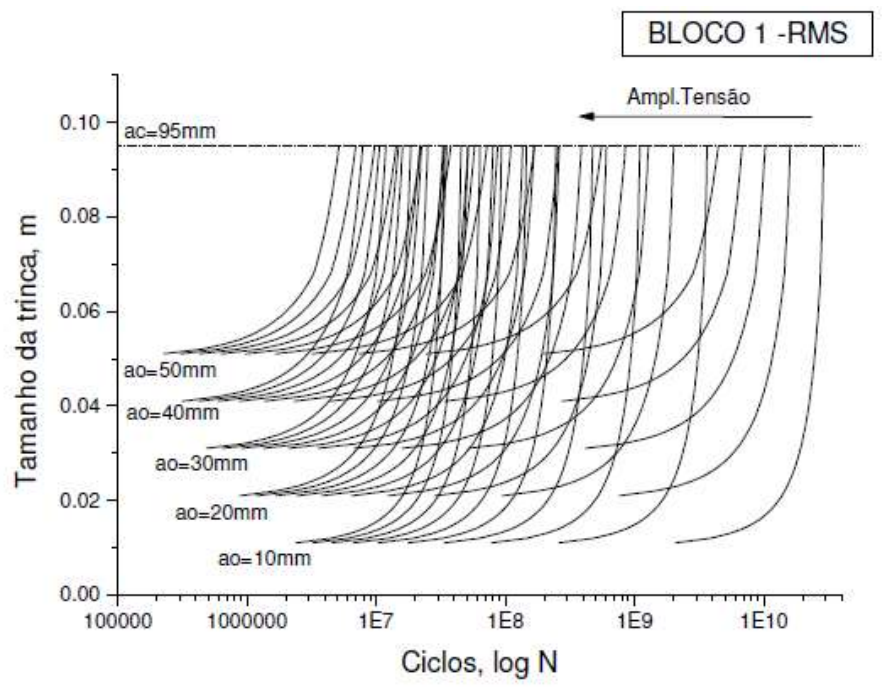

Fonte: Autores (2021).

Figura 7 - Tamanho da trinca x Número de ciclos para diversos comprimentos iniciais de trincas - BLOCO 6 de carregamentos.

\section{BLOCO 6 - RMS}

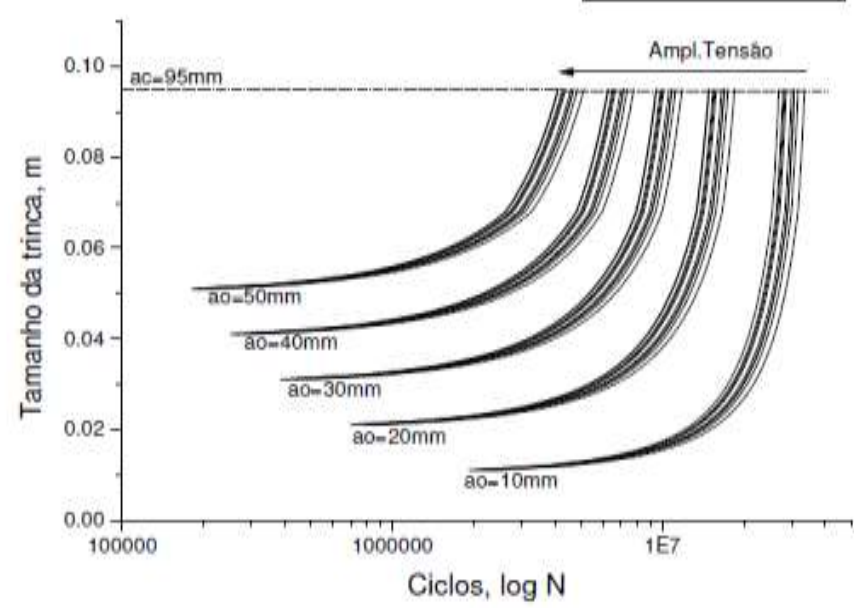

Fonte: Autores (2021).

Analisando o comportamento do crescimento das trincas através do gráfico plotado, observa-se uma tendência natural da vida da estrutura diminuir com o aumento do tamanho das trincas iniciais e com o aumento da amplitude de tensão RMS $\left(D s_{r m s}\right)$. Observa-se também que trincas de tamanhos diferentes podem levar o mesmo tempo para crescer até um valor $\mathrm{a}_{\mathrm{c}}$ se estiverem sujeitas a níveis de amplitudes de tensões diferentes.

Outro gráfico que podem ajudar a obter conclusões são de vida da estrutura versus amplitude de tensão, mostrados na Figura 7 e Figura 8. Através destes gráficos observa-se que quanto maior a amplitude de tensão menor é a dispersão dos resultados de vida da estrutura para diferentes comprimentos iniciais de trincas. 
Figura 8 - Vida da estrutura x Amplitude de tensão RMS para diferentes tamanhos iniciais de trincas - BLOCO 1.

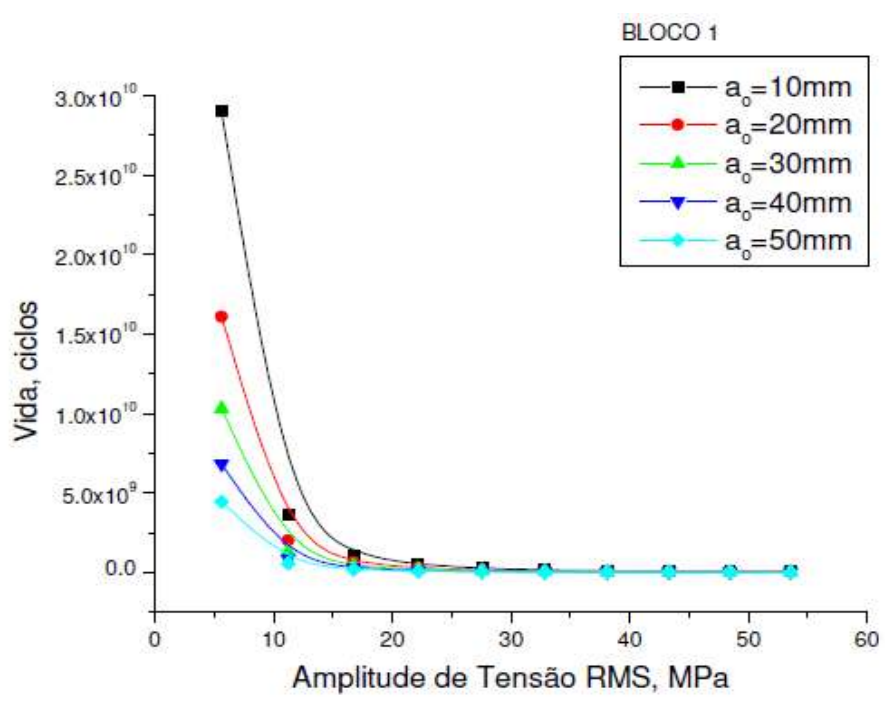

Fonte: Autores (2021).

Figura 9 - Vida da estrutura x Amplitude de tensão RMS para diferentes tamanhos iniciais de trincas - BLOCO 6.

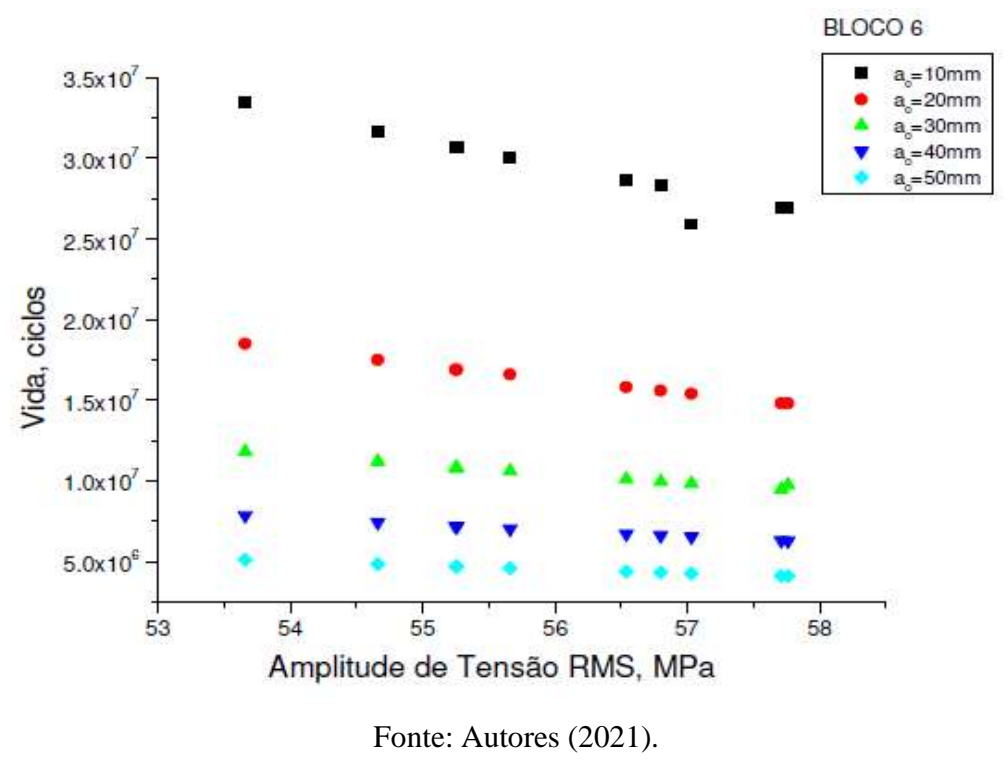

Visualmente observa-se que acima de um valor da amplitude de tensão, em torno de $20 \mathrm{MPa}$, a dispersão ou desvio padrão dos resultados de vida da estrutura para diferentes tamanhos de trincas iniciais tende a permanecer constante. Este fato pode ser melhor visualizado na Figura 9, correspondente ao BLOCO 6 de carregamentos, onde as amplitudes de tensão estão na faixa de $50 \mathrm{MPa}$ a $60 \mathrm{MPa}$. Observa-se também uma menor dispersão para tamanhos maiores de trincas iniciais.

\subsection{Resultados de Simulações com o Modelo Ciclo-a-Ciclo}

Com a utilização do modelo Ciclo-a-Ciclo nas simulações de crescimento das trincas observou-se um grande esforço computacional para o cálculo de vida da estrutura. O modelo apresenta as vantagens de garantir a inatividade da trinca quando num ciclo $D K_{i}<D K_{\text {th }}$ e prever a fratura súbita causada por um grande pico durante o carregamento variável quando $K_{\max }<K_{\epsilon}$; por outro lado, um aumento na precisão dos resultados não justifica a utilização do modelo, quando em comparação com o modelo RMS. 
Desta forma, utiliza-se o modelo Ciclo-a-Ciclo para prever a inatividade das trincas ou fratura súbita causada por sobrecargas. Considerou-se uma quantidade de 5.000 ciclos iniciais de carregamentos, onde a inatividade da trinca ocorre quando a variação do fator de intensidade de tensão no ciclo $D K_{\tilde{i}}$ é inferior ao valor de $D K_{t h}$ mínimo necessário para que aconteça a propagação.

Analisando as taxas de crescimento das trincas para os diferentes BLOCOS de carregamento, observa-se que para determinados níveis de tensão e tamanhos iniciais de trincas, estas permanecem inativas. Isto sugere que num caso real de defeito numa estrutura, como uma viga de ponte, pode-se fazer uma análise de tensões locais e restringir o tráfego a determinados níveis de tensão para que não haja progresso da trinca e garantir um tempo conveniente de reparo.

Além disso, as taxas de propagações de trincas estão dentro da região de Paris, iniciando a propagação com taxas $d A / d N$ na ordem de ${ }^{10^{-10}} \mathrm{~m} /$ ciclo . Não se observou fratura súbita nos primeiros 5.000 ciclos, o que não garante que uma ruptura repentina não possa acontecer posteriormente, pois à medida que a trinca avança o fator de intensidade tensões aumentam sua magnitude.

Na Figura 10, observa-se o comportamento do crescimento da trinca durante 200 ciclos para os diferentes tipos de carregamentos do BLOCO 6, onde estes são todos aleatórios.

Figura 10 - Crescimento de trincas com $\boldsymbol{a}_{\mathbf{0}}=\mathbf{1 0} \mathbf{m m}$ sujeitas ao BLOCO 6 de carregamentos.

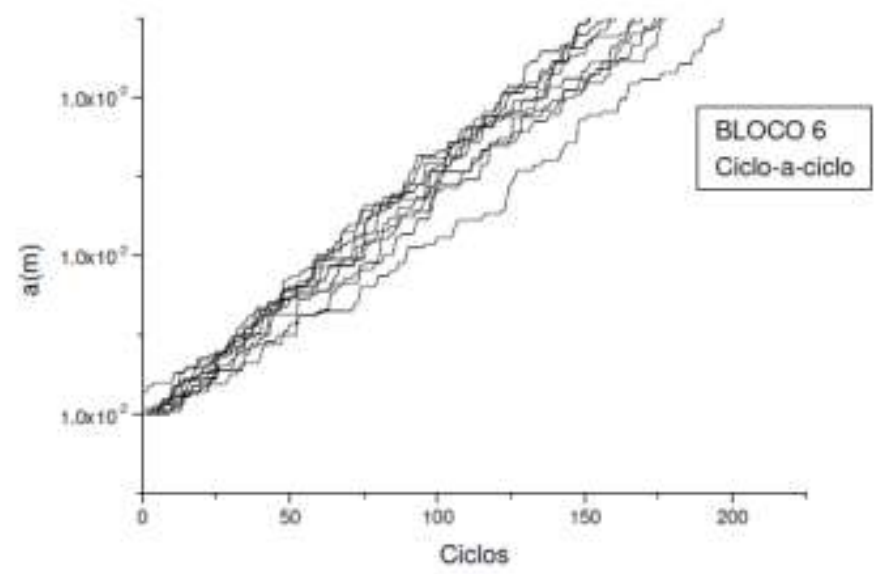

Fonte: Autores (2021).

\section{Conclusão}

Desenvolveu-se neste trabalho um modelo computacional de propagação de trincas, utilizando-se a teoria do Valor Médio Quadrático e Ciclo-a-Ciclo. Estes apresentaram resultados satisfatórios na análise de propagação de trincas quando comparados com um programa de reconhecida precisão.

Verificou-se durante as simulações que o modelo do Valor Médio Quadrático (RMS) possui a vantagem de ser computacionalmente mais eficiente em relação ao Ciclo-a-Ciclo, porém este não prevê a inatividade da trinca para baixos valores do fator de intensidade de tensão ou ruptura súbita por sobrecarga.

O trabalho apresenta uma metodologia de previsão de vida da estrutura que pode ser adaptada a outros tipos de elementos estruturais com as devidas mudanças relativas às suas particularidades.

\section{Agradecimentos}

A CAPES e ao CNPq pelo financiamento da pesquisa. 


\section{Referências}

Arias, A. R., \& Bracarense, A. Q. (2015). Velocidade de propagação de trinca por fadiga de soldas subaquáticas molhadas: avaliação fora da água. Soldagem \& Inspeção, 20(4), 403-411.

Chen, S. R., \& Wu, J. (2010). Dynamic performance simulation of long-span bridge under combined loads of stochastic traffic and wind. Journal of Bridge Engineering, 15(3), 219-230.

Dantas, J. P. R. (2010). Investigação experimental da fadiga em lajes de pontes armadas com barras ou telas soldadas (Doctoral dissertation, Universidade de São Paulo).

Deus, E. P. D. (1997). Análise do processo de fraturamento em vigas de pontes de aço sob efeito de fadiga (Doctoral dissertation, Universidade de São Paulo).

Ding, Y., Wu, D., Su, J., Li, Z. X., Zong, L., \& Feng, K. (2021). Experimental and numerical investigations on seismic performance of RC bridge piers considering buckling and low-cycle fatigue of high-strength steel bars. Engineering Structures, 227, 111464.

Garcez, M. R., Silva Filho, L. C. P., \& Meier, U. (2012). Reforço de vigas de concreto armado com laminados de PRFC protendidos: parte 2: análise sob ação de carregamento cíclico. Revista IBRACON de Estruturas e Materiais, 5(4), 420-439.

Grzeskowiak, R. M., Freeman, L. R., Harper, D. P., Anderson, D. E., \& Mulon, P. Y. (2021). Effect of cyclic loading on the stability of screws placed in the locking plates used to bridge segmental bone defects. Journal of Orthopaedic Research®, 39(3), 516-524.

Haghani, R., \& Yang, J. (2016). Application of FRP materials for construction of culvert road bridges: manufacturing and life-cycle cost analysis. Rapport, (2016: 3).

Huang, X., Torgeir, M., \& Cui, W. (2008). An engineering model of fatigue crack growth under variable amplitude loading. International Journal of Fatigue, 30(1), 2-10.

Komarovsky, A. A., \& Haslach Jr, H. W. (2003). Physics of strength and fracture control: adaptation of engineering materials and structures. Appl. Mech. Rev., 56(5), B65-B66.

Kujawski, D. (2001). A fatigue crack driving force parameter with load ratio effects. International Journal of Fatigue, 23, $239-246$.

Kwon, K., \& Frangopol, D. M. (2010). Bridge fatigue reliability assessment using probability density functions of equivalent stress range based on field monitoring data. International journal of fatigue, 32(8), 1221-1232.

Law, S. S., \& Zhu, X. Q. (2004). Dynamic behavior of damaged concrete bridge structures under moving vehicular loads. Engineering Structures, 26(9), 12791293 .

Lu, P., Zhou, C., Huang, S., Shen, Y., \& Pan, Y. (2021). Experimental Study on Mix Ratio Design and Road Performance of Medium and Small Deformation Seamless Expansion Joints of Bridges. Transportation Research Record, 0361198120984741.

Manhart, J., Kunzelmann, K. H., Chen, H. Y., \& Hickel, R. (2000). Mechanical properties of new composite restorative materials. Journal of Biomedical Materials Research: An Official Journal of The Society for Biomaterials, The Japanese Society for Biomaterials, and The Australian Society for Biomaterials and the Korean Society for Biomaterials, 53(4), 353-361.

Pu, Q., Yang, S., Shi, Z., Hong, Y., \& Zhou, Y. (2021). Fatigue Performance of an Innovative Steel-Concrete Joint in Long-Span Railway Hybrid Box Girder Cable-Stayed Bridges. Journal of Bridge Engineering, 26(2), 04020129.

Pereira, A. S. et al. (2018). Metodologia da pesquisa científica. https://repositorio.ufsm.br/bitstream/handle/1/15824/Lic_Computacao_Metodologia-PesquisaCientifica.pdf?sequence $=1$.

Skorupa, M. (1998). Load interaction effects during fatigue crack growth under variable amplitude loading-a literature review. Part I: empirical trends. Fatigue \& Fracture of Engineering Materials \& Structures, 21(8), 987-1006.

Terasawa, T., Akimoto, M., Nishi, H., \& Komuro, M. (2021). Cyclic Loading Tests on Seismic Retrofit of Reinforced Concrete Bridge Pier with Embedded Seismic Retrofit Rebar and Aramid Fiber-Reinforced Plastic Sheets Jacketing. In EASEC16 (pp. 781-790). Springer, Singapore.

Wang, W. Y., Li, P., Lin, D., Tang, B., Wang, J., Guan, Q., \& Liu, W. (2020). DID Code: A Bridge Connecting the Materials Genome Engineering Database with Inheritable Integrated Intelligent Manufacturing. Engineering, 6(6), 612-620. 